\title{
Can a standardized plant immunomodulator (rice bran arabinoxylan concentrate/MGN-3) increase the effects of MEK and BRAF inhibitors with clinical benefit? Case report of a patient with carcinoma in biliary duct
}

Tibor Hajto*

Medical University Pecs, Hungary

\begin{abstract}
Background: Targeting hyperactive mitogen-activated protein kinase (MAPK) signaling cascade has proven to be an effective treatment for a variety of different cancers. Using an important member of this cascade, namely MEK (mitogen activated extracellular signal regulation kinase) inhibitors, the clinical responses are often transient and complete remission is rarely observed. Outgrowth of resistant clones within progressed tumors appears to be inevitable. Recent immunological and clinical observations suggest that in background of this resistance the tumor-.induced disturbance of immunoregulation at least in part may play a role. Namely, it was shown that growth factor receptor signaling pathway inhibitors can increase the immune sensitivity of tumor cells, but they can't activate the down regulated immune effectors. Consequently, the combination of MAPK cascade signaling pathway inhibitors and the immune effectors activating immunomodulators may be a promising new strategy.
\end{abstract}

Material and methods: In a now 59 years old patient with inoperable (BRAF-mutant) low differentiated adenocarcinoma of bilary ducts after 30GY radiotherapy and two cycles (Gemcitabin+ Cisplatin) chemotherapy a rapid progression of lung, liver and brain metastases were by CT and MR established. Thereafter, a teatment with BRAF+MEK inhibitors (2x150 mg dabrafenib and 1 x $2 \mathrm{mg}$ trametinib) was started. These inhibitors were combined with daily $45 \mathrm{mg} / \mathrm{kg}$ rice bran arabinoxylan concentrate (using Biobran/MGN-3) which was shown to be a pathogenic associated molecular pattern (PAMP)-like molecule and can stimulate the type-1 innate immune cells against tumor cells. Results: After the chemotherapy and prior to the start of second line treatment, the patient had a nearly terminal state of her rapidly progressive disease. Eight months after the combination of MEK / BRAF inhibitor and immunomodulator therapy nearly complete remissions of all metastases was established in CT and MR.

Conclusion: This case report may support a hypothesis that MEK/ BRAF inhibitors and type-1 immune cells activating immunomodulators together may synergistic inhibit the tumor growth. Further clinical investigations are necessary to clarify this question.

\section{Introduction}

MEK (Mitogen activated Extracellular signal regulation Kinase) - inhibitors can down regulate the receptor tyrosine kinase mediated signaling pathway by inhibition of mitogen activated protein kinase /MAPK/ cascade (RAS/RAF/MEK/ERK) which regulates cell proliferation, cell cycle progression and cell migration. Dysregulation of the MAPK cascade occurs in more than one-third of all malignancies, a process that has fostered the development of targeted MAPK pathway inhibitors [1]. Owing to the wide spread activation of this pathway in numerous neoplasms, MEK inhibitors are being studied in combination with other targeted and cytotoxic drugs in a variety of clinical situations [2-4]. MEK inhibitory compounds demonstrated markedly enhanced potency against BRAF (V600E) cancer cells compared to cell lines lacking this oncogenic pathway mutation. In addition, between MEK and BRAF inhibitors exhibit a synergistic effect [5].

Despite the improved outcomes with monoclonal antibodies targeting various members of the MAPK cascade, durable clinical responses are uncommon. Outgrowth of resistant clones within progressed tumors appears inevitable [6]. Recent immunological and clinical observations suggest that in background of this resistance at least in part the tumor-induced disturbance of immunoregulation may play also a role. In spite of an enhanced sensitivity of tumor cells for immunological killing by targeted MAPK pathway inhibitors [7-8] the most important immune effector cells against tumor are parallel not activated.

As it well known, tumor cells are definitively able to escape from the $T$ cell lyse since they exhibit genetic dysregulation related irreversible quantitative and qualitative alterations of their MHC-I antigens [9]. Consequently, growing attention is focusing on the mechanisms of innate immune system which are able to kill tumor cells in a nonMHC-restricted manner and appear to exhibit more reversible escape mechanisms if it is compared with adoptive system. As known, the effector cells of innate immune system are committed in two directions. Type-1 macrophages (M1) and from the monocytes originating type-1 dentritic cells (DC1) generate (in a rigorously regulated cascade system

Correspondence to: Tibor Hajto, Professor, Medical University Pecs, Hungary, Tel. +36-309-735-337; E-mail: drhajtot@t-online.hu

Key words: MEK-inhibitor, dabrafenib, BRAF-inhibitor, trametinib, immunomodulatory treatment, tumor disease, rice bran arabinoxylan, MGN-3

Received: August 23, 2017; Accepted: September 25, 2017; Published: September 27,2017 
Hajto T (2017) Can a standardized plant immunomodulator (rice bran arabinoxylan concentrate/MGN-3) increase the effects of MEK and BRAF inhibitors with clinical benefit? Case report of a patient with carcinoma in biliary duct

only for short time) proinflammatory cytokines, IL-12 and activate cytotoxic effector (such as NK and NKT) cells which are potent inhibitors of tumor growth. However, these type-1 cells are defective in tumor patients. Available information suggests that tumor-associated macrophages belong to the prototypic M2 population [10,11]. M2 macrophages and from the plasmocytoid precursors originating DC2 dentritic cells generate IL-4 and IL-10 which facilitate the generation of Th2 cells and inhibit Th1 cells and the type-1 natural system [10]. M2 macrophages and CD2 dentritic cells affect chronic inflammation, promote cell proliferation by producing growth factors and stimulate angiogenesis. Tumor patients can have up to $40 \%$ more M2 peripheral monocytes than healthy individuals who have only $10 \%$ M2 monocytes $[10,11]$.

As it was mentioned, the dominance of $\mathrm{M} 2$ and $\mathrm{CD} 2$ cells in tumor patients can lead to an enhanced production of growth factors which are also contribute to the down regulation of the type-1 natural immune cells. It was shown that growth factors can inhibit the NK cytotoxicity against cancer cells by down regulating the expression of the so-called stress- related receptors on tumor cell membrane, namely the Killing Activator Receptor (KAR) binding NKG2D ligands: UL-16 binding proteins (ULBP1 and ULBP2) or MICA and MICB [7,8]. Taken together these observations, we can conclude that targeted MAPK pathway inhibitors can enhance the sensitivity of tumor cells against immune effectors but they are not able to activate them. Therefore, the hypothesis is arisen as to whether immunomodulators, which are able to activate the (type-1) immune effectors against tumor cells, may improve the effects of the targeted MAPK pathway inhibitors. In this paper, a case report is presented which may support this hypothesis. A patient with inoperable (BRAF-mutant) low differentiated adenocarcinoma of bilary ducts with lung, liver and brain metastases was treated with a combination of MEK and BRAF inhibitors together with evidence based and standardized plant immunomodulator (BioBran/MGN-3) which was shown to stimulate the type-1 natural immune cells. After four months all metastases showed a nearly complete remission which were after 8 months also established.

\section{Material and methods}

\section{MEK and BRAF inhibitors}

As MEK inhibitor $1 \times 2 \mathrm{mg}$ trametinib and as BRAF inhibitor $2 \times 150$ mg dabrafenib were given from July $4^{\text {th }} 2016$ regularly until March 2017 (observation period). The patient was regularly controlled by $\mathrm{CT}$ and MR investigations by the Oncological Department of Medical University Debrecen (Hungary).

\section{Standardized rice bran extract (BioBran/MGN-3)}

The immunomodulatory drug used in the combinative treatment of the presented patients is BioBran/MGN-3 which is manufactured and supplied by Daiwa Pharmaceutical Co, Ltd, Tokyo, Japan. BioBran/ MGN-3 is composed of denaturated hemicellulose, which is obtained by rice bran hemicellulose reacting with multiple carbohydratehydrolyzing enzyme from shiitake mushrooms. BioBran/MGN-3 is standardized for its main chemical component: arabinoxylan with a xylose (in its main chain) and with an arabinose polymer (in its side chain). To the presented patient BioBran/MGN-3 was given orally in doses of $45 \mathrm{mg} / \mathrm{kg}$ at first daily and four months later three times a week.

\section{Ethics committee}

Ethics committee proposed to observe and publish case reports of own patients treated with standardized plant immunomodulators. The patient has given an informed consent to process and publish her dates.
This case report may stimulate an interest for other research groups according to the opinion of the ethics committee.

\section{Results}

A now 59 year old patient with inoperable low differentiated adenocarcinoma of biliary ducts had in April 2016 a terminal stage of her disease. By PET/CT investigations multiplex metastases were established in the following organs: lung (S3), bilateral mediastinal, paraaortal, paratracheal, subcarinal, subclavicular lymph nodes, pancreas, hilus of liver and mesenterium. In addition, four brain metastases (three in cerebellum and one in temporal region) were detected by MR investigation. After an irradiation (30 GY WBRT), in Mai/June 2016 two cycles Cisplatin and Gemcitabin were given. At end of June 2016 progressions of all metastases were established and therefore the chemotherapy was stopped. Since by the genetic investigation 15\% BRAF (V600E) clinical relevant driver mutation and $22 \%$ TP53 $\left(\mathrm{Q} 16 \mathrm{fs}^{\star} 28\right)$ clinical relevant driver mutation were found, at begin of July $20161 \times 2 \mathrm{mg}$ trametinib MEK inhibitor and $2 \times 150 \mathrm{mg}$ dabrafenib BRAF inhibitor were given. At the same time an immunomodulatory treatment was started with a standardized bran rice arabinoxylan concentrate (BioBran/MGN-3) in doses of $3 \mathrm{~g}$ daily for four months and thereafter three times $3 \mathrm{~g}$ a week. During this combinative therapy a very rapid remission of all metastases were observed. Figures 1 and 2 show the rapid decreases of tumor markers (CEA and CA19-9) in peripheral blood. After four and eight months nearly complete remissions (CR) of all metastases were also established by $C T$ and MR investigations. Figure 3 represents a CR of a brain metastasis prior the treatment and eight months later.

\section{Discussion}

The effect of the immune system on the inhibitory targeting therapy of the tyrosin kinase mediated signaling pathway is poorly understood since very few research results are now available. Although signaling pathway are usually depicted as linear cascades, nearly all signaling pathway are highly interconnected and form networks that allow dynamic regulation of the timing, strength and duration. In addition, both feed forward and feed backward loops provide the means to self-regulation signals. As a consequence, the same signal may induce different outcomes depending on the particular cell state. Consequently, they can inhibit or stimulate in different cells [6]. Interestingly, it

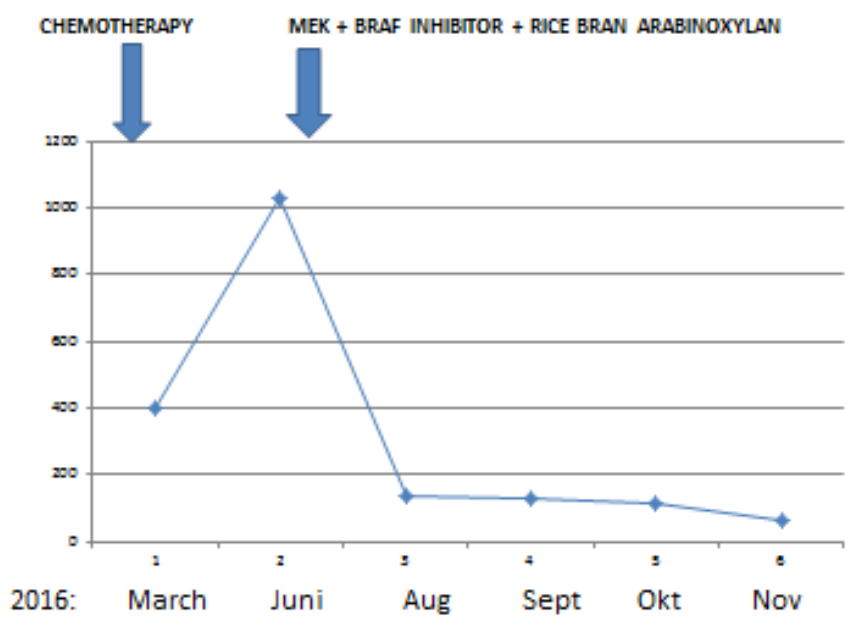

Figure 1. Rapid improvement of CA19-9 tumor marker after the start of a treatment with $1 \times 2 \mathrm{mg}$ trametinib (MEK inhibitor) and 2x150 mg (dabrafenib BRAF inhibitor) combined with daily $45 \mathrm{mg} / \mathrm{kg}$ standardized rice bran arabinoxylan immunomodulator (Biobran). 
Hajto T (2017) Can a standardized plant immunomodulator (rice bran arabinoxylan concentrate/MGN-3) increase the effects of MEK and BRAF inhibitors with clinical benefit? Case report of a patient with carcinoma in biliary duct

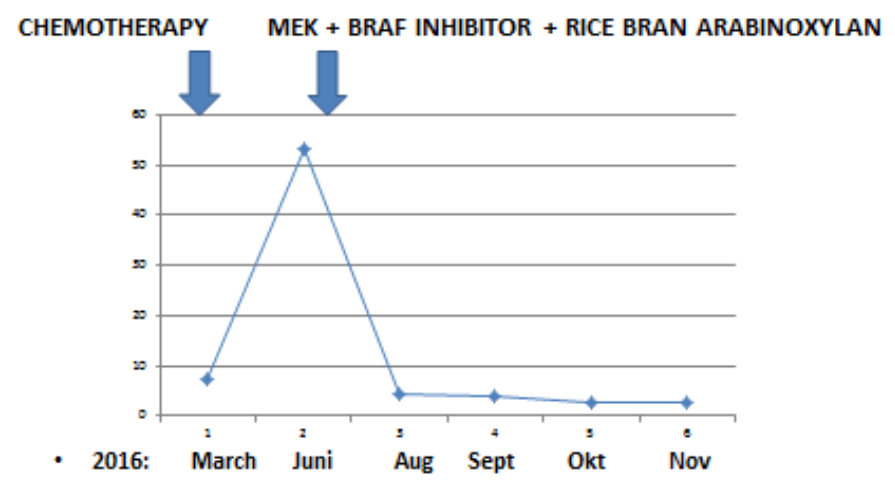

Figure 2. Rapid improvement of CEA tumor marker after the start of a treatment with $1 \times 2$ $\mathrm{mg}$ trametinib (MEK inhibitor) and 2x150 mg dabrafenib (BRAF inhibitor) combined with daily $45 \mathrm{mg} / \mathrm{kg}$ standardized rice bran arabinoxylan immunomodulator (Biobran).
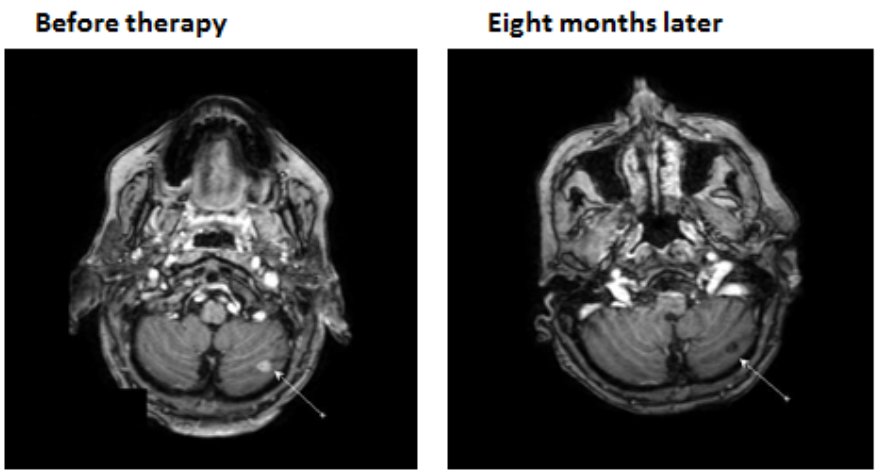

Figure 3. MR investigations represent a complete remission of a brain metastasis (in cerebellum) after a treatment with $1 \times 2 \mathrm{mg}$ trametinib (MEK inhibitor) and $2 \times 150 \mathrm{mg}$ dabrafenib (BRAF inhibitor) combined with $45 \mathrm{mg} / \mathrm{kg}$ standardized rice bran arabinoxylan immunomodulator (Biobran).

appears to be a similarity between the regulation of MAPK cascade and the regulation of natural immunity. As it was mentioned above, the effector cells of innate immune system are also committed in two directions $[10,11]$ which also in cascade system can play an important role in the activation and inhibition of cell proliferation. Both protein kinase signaling pathway and innate immunity exist in a basal state and they are activated only as necessary by divergent regulation stimuli $[12,13]$. The mechanisms for switching between dormant and activate state in both systems can be intricate, but it is probable that both signaling pathways are highly interconnected and form networks. Therefore, it is not surprising that growth factors stimulate the cell proliferation on the one hand and protect the cells (down regulating their ligands) against immune effectors on the other hand inducing a decrease in tumor defense. Consequently, a combination of protein kinase signaling pathway inhibitors and natural effector cells activating immunomodulators may open new perspectives in the tumor therapy. This case report may support this hypothesis. However, in the future we must better learn to manipulate in the regulatory networks, and the timing, strength and duration of responses must be taken with more attention into consideration. For example, the stronger a response, the less possible to give a drug daily, since therapy free intervals can be necessary $[13,14]$.

Pathogenic Associated Molecular Pattern (PAMP) molecules are the best candidates for this combination of targeting therapy with immunomodulation. However, PAMP molecules exist only in the nature (bacteria or plants). The chemistry is not able to produce these configurations. Bacteria have toxic side effects but plants have not. Unfortunately, the best evidence based and standardized immunodulators without any side effects are plant extracts and they are all over the world registered only as food supplements. There are growing evidences that plant PAMP-like molecules (such as standardized rice bran arabinoxylan concentrate) exhibit a lot of immunological [15-26] and clinical benefit [27-29]. Therefore, further clinical trials are necessary to investigate this beneficial interaction.

\section{Disclosure statement}

The authors declare that there is no competing or other conflicting interest in relation to this paper.

\section{References}

1. Roskoski R Jr (2017) Allosteric MEK1/2 inhibitors including cobimetanib and trametinib in the treatment of cutaneous melanomas. Pharmacol Res 117: 20-31. [Crossref]

2. Li L, Zhao GD, Shi Z, Qi LL, Zhou LY, et al. (2016) The Ras/Raf/MEK/ERK signaling pathway and its role in the occurrence and development of HCC. Oncol Lett 12: 30453050. [Crossref]

3. Chaparro M, González Moreno L, Trapero-Marugán M, Medina J, Moreno-Otero R (2008) Pharmacological therapy for hepatocellular carcinoma with sorafenib and other oral agents. Aliment Pharmacol Ther 28: 1269-1277. [Crossref]

4. Stinchcombe TE, Johnson GL (2014) MEK inhibition in non-small cell lung cancer. Lung Cancer 86: 121-125. [Crossref]

5. Solit DB, Garraway LA, Pratilas CA, Sawai A, Getz G, et al. (2006) BRAF mutation predicts sensitivity to MEK inhibition. Nature 439: 358-362. [Crossref]

6. Smith MP, Wellbrock C (2016) Molecular pathways: Maintaining MAPK inhibitor sensitivity by targeting nonmutational tolerance. Clin Cancer Res 22: 5966-5970. [Crossref]

7. Bae JH, Kim SJ, Kim MJ, Oh SO, Chung JS, et al. (2012) Sussceptibility to natural killer cell-mediated lysis of colon cancer cells is enhanced by treatment with epidermal growth factor receptor inhibitors through UL16 binding protein-1 induction. Cancer Sci 103: 7-16. [Crossref]

8. He S, Yin T, Li D, Gao X, Wan Y, et al. (2013) Enhanced interaction between natura killer cells and lung cancer cells: involvement in gefitinib-mediated immunoregulation. $J$ Transl Med 11: 186. [Crossref]

9. Venza M, Visalli M, Catalano T, Beninati C, Teti D, Venza I (2017) Epidrugs in the immunotherapy of cutaneous and uveal melanoma. Anticancer Agents Med Chem 17: 190-205. [Crossref]

10. Mantovani A (2007) Inflammation and cancer; the macrophage connection. Medicina (Buenos Aires) 67: 32-34.

11. Sánchez-Torres C, García-Romo GS, Cornejo-Cortés MA, Rivas-Carvalho A, SánchezSchmitz G (2001) CD16+ and CD16- human blood monocyte subsets differentiate in vitro to dentritic cells with different abilities to stimulate CD4+ T cells. Int Immunol 13: 1571-1581. [Crossref]

12. Roskoski R Jr (2015) A historical overview of protein kinases and their targeted small molecule inhibitors. Pharmacol Res 100: 1-23. [Crossref]

13. Hajto T, Fodor K, Perjési P (2009) Difficulties and perspectives of immunomodulatory therapy with mistletoe lectins and standardized mistletoe extracts in evidence based medicine. eCAM 2009; 1-6.

14. Ali KH, Mellilo AB, Leonard SM, Asthana D, Woolger JM, et al. (2012) An openlabel, randomized clinical trial to assess the immunomodulatory activity of a nove oligosaccharide compound in helthy adults. Functional Foods Health Disease 2: 265-279.

15. Cholujova D, Jakubikova J, Czako B, Martisova M, Hunakova L, et al. (2013) MGN 3 arabinoxylan rice bran modulates innate immunity in multiple myeloma patients. Cancer Immunol Immunother 62: 437-445.

16. Cholujova D, Jakubikova J, Sedlak J (2009) BioBran-augmented maturation of human monocyte-derived dendritic cells. Neoplasma 56: 89-95. [Crossref]

17. Tan BL, Norhaizan ME (2017) Scientific evidence of rice by-products for cancer prevention: chemopreventive properties of waste products from rice milling on carcinogenesis in vitro and in vivo. Biomed Res Int 2017: 9017902.

18. Pérez-Martínez A, Valentín J, Fernández L, Hernández-Jiménez E, López-Collazo E, et al. (2015) Arabinoxylan rice bran (MGN-3/Biobran) enhances natural killer cellmediated cytotoxicity against neuroblastoma in vitro and in vivo. Cytotherapy 17: 601612. [Crossref] 
Hajto T (2017) Can a standardized plant immunomodulator (rice bran arabinoxylan concentrate/MGN-3) increase the effects of MEK and BRAF inhibitors with clinical benefit? Case report of a patient with carcinoma in biliary duct

19. Badr El-Din NK, Ali DA, Alaa El-Dein M, Ghoneum M (2016) Enhancing the apoptotic effect of a low dose of paclitaxel on tumor cells in mice by arabinoxylan rice bran (MGN-3/Biobran). Nutr Cancer 68: 1010-1020. [Crossref]

20. Badr El-Din NK, Abdel Fattah SM, Pan D, Tolentino L, Ghoneum M. Chemopreventive activity of MGN-3/Biobran against chemical induction of glandular stomach carcinogenesis in rats and its apoptotic effect in gastric cancer cells. Integr Cancer Ther 15: NP26-NP34. [Crossref]

21. Ghoneum M, Agrawal S (2014) Mgn-3/biobran enhances generation of cytotoxic CD8+ $\mathrm{T}$ cells via upregulation of dec-205 expression on dendritic cells. Int J Immunopathol Pharmacol 27: 523-530. [Crossref]

22. Ghoneum M, Badr El-Din NK, Ali DA, El-Dein MA (2014) Modified arabinoxylan from rice bran, MGN-3/biobran, sensitizes metastatic breast cancer cells to paclitaxel in vitro. Anticancer Res 34: 81-87. [Crossref]

23. Ghoneum M, Badr El-Din NK, Abdel Fattah SM, Tolentino L (2013) Arabinoxylan rice bran (MGN-3/Biobran) provides protection against whole-body ?-irradiation in mice via restoration of hematopoietic tissues. J Radiat Res 54: 419-429. [Crossref]

24. Ghoneum M, Agrawal S (2011) Activation of human monocyte-derived dendritic cells in vitro by the biological response modifier arabinoxylan rice bran (MGN-3/Biobran) Int J Immunopathol Pharmacol 24: 941-948. [Crossref]

25. Badr El-Din NK, Noaman E, Ghoneum M (2008) In vivo tumor inhibitory effects of nutritional rice bran supplement MGN-3/Biobran on Ehrlich carcinoma-bearing mice. Nutr Cancer 60: 235-244. [Crossref]

26. Gollapudi S, Ghoneum M (2008) MGN-3/Biobran, modified arabinoxylan from rice bran, sensitizes human breast cancer cells to chemotherapeutic agent, daunorubicin. Cancer Detect Prev 32: 1-6. [Crossref]

27. Bang MH, Riep TV, Thinh NT, Song LH, Dung TT, et al. (2010) Arabinoxylan rice bran (MGN-3) enhances the effect of interventional therapies for the treatment of hepatocellular carcinoma: A three-year rabdomized clinical trial. Anicancer Res 30: 5145-5152. [Crossref]

28. Itoh Y, Mizuno M, Ikeda M, Nakahara R, Kubota S, et al. (2015) A randomized, doubleblind pilot trial of hydrolyzed rice bran versus placebo for radioprotective effect on acute gastroenteritis secondary to chemoradiotherapy in patients with cervical cancer. Evid Based Complement Alternat Med 2015: 974390.

29. McDermott C, Richards SC, Thomas PW, Montgomery J, Lewith G (2006) A placebocontrolled, double-blind, randomized controlled trial of a natural killer cell stimulant (BioBran MGN-3) in chronic fatigue syndrome. QJM 99: 461-468. [Crossref]

Copyright: $(02017$ Hajto T. This is an open-access article distributed under the terms of the Creative Commons Attribution License, which permits unrestricted use, distribution, and reproduction in any medium, provided the original author and source are credited. 